\title{
Credibility of Scientific Information on Social Media: Variation by Platform and Presence of Formal Credibility Cues
}

\author{
Clara Boothby ${ }^{1 *}$, Dakota Murray ${ }^{1}$, Anna Polovick Waggy ${ }^{1}$, Andrew Tsou ${ }^{1}$, Cassidy R. Sugimoto ${ }^{1}$
}

${ }^{1}$ School of Informatics, Computing, and Engineering, Indiana University Bloomington, Bloomington, Indiana, United States of America

\begin{abstract}
Responding to calls to take a more active role in communicating their research findings, scientists are increasingly using open online platforms, such as Twitter, to engage in science communication or to publicize their work. Given the ease at which misinformation spreads on these platforms it is important for scientists to present their findings in a manner that appears credible. To examine the extent to which the online presentation of science information relates to its perceived credibility, we designed and conducted two surveys on Amazon's Mechanical Turk. In the first survey, participants rated the credibility of science information on Twitter compared with the same information other platforms, and in the second, participants rated the credibility of tweets with modified characteristics: presence of an image, text sentiment, and the number of likes/retweets. We find that similar information about scientific findings is perceived as less credible when presented on Twitter compared to other platforms, and that perceived credibility increases when presented with recognizable features of a scientific article. On a platform as widely distrusted as Twitter, use of these features may allow researchers who regularly use Twitter for research-related networking and communication to present their findings in the most credible formats.
\end{abstract}

\section{Keywords}

Science Communication; Credibility; Twitter; Platform; Scholarly Communication; Social Media 


\section{Introduction}

1

2

3

4

Scientific institutions and scientists themselves are increasingly making use of online communication platforms to disseminate scientific findings (Duggan et al., 2015). Covering science in informal outlets, such as Twitter, alongside traditional science news and peer-reviewed journal articles has the potential to reach a cross-section of science enthusiasts (Büchi, 2016; Ranger \& Bultitude, 2014). However, presented with this crowded online arena, readers will employ various heuristics, conscious and unconscious, to assess the credibility of information (Flanagin \& Metzger, 2007). Here, we define credibility as a perceived feature of trustworthiness that readers apply to information they encounter (Schmierbach \& Oeldorf-Hirsch, 2012). If writers and publishers of online science information want information they present to be seen as credible, they will need to be cognizant of the methods users employ to make these judgements. In this study we focus on describing features, of both platforms and text, that contribute to user perceptions of the credibility of science information when presented online. How and where scientists present their work may enhance or undermine its credibility, and a stronger understanding of these features is important to upholding the credibility of reliable science information, particularly in platforms that struggle to filter out misinformation.

The reasons for science journalists to present their work online is self-evident, but there are also many reasons for scientists to present their findings online. In addition to science outreach, many researchers find general-use social media applications useful during many stages of research, including selecting research questions, collaborating, publicizing findings, and keeping up to date on recent publications (Collins et al., 2016; Côté \& Darling, 2018; Holmberg et al., 2014; Rowlands et al., 2011; Tenopir et al., 2013). The utility of general use social media emerges in part from their lower time demands and barriers to entry than more traditional forms of outreach (McClain, 2017), and Twitter in particular has been a major platform used by scientists for outreach (Côté \& Darling, 2018; Mohammadi et al., 2018). 
The use of Twitter among scientists also appears to be increasing, as the majority of those users

24 reported having their accounts for less than two years (Collins et al., 2016). While the choice of whether or not to engage in informal online science communication has been the subject of some controversy (Collins et al., 2016; Yammine et al., 2018), there is less guidance about selecting between the myriad of

27 platforms for online engagement or about effectively composing posts. As many researchers are poised to engage in social media as a professional task, the stakes are high for maintaining the credibility of the information they present online.

Credibility is understood as a subjective feature that is perceived by individual readers and not an innate feature of the information (Bian, 2012; Schmierbach \& Oeldorf-Hirsch, 2012; Shariff et al., 2017; Tseng \& Fogg, 1999). As such, online credibility evaluations are commonly understood to be influenced by formal cues, the features surrounding the presentation of information, such as website design, source attribution, and genre conventions (Flanagin \& Metzger, 2007). Even within a single online platform, differences in writing style and narrative frame (Shariff et al., 2017), cues indicating audience reception (Winter \& Krämer, 2014), and the perception of both scientific and social consensus (Kobayashi, 2018) features on credibility, specific media platforms often have strong reputations among users that affect their perceptions of the information on those platforms (Lucassen \& Schraagen, 2013; Winter \& Krämer, 2014). While it would be challenging to disentangle a platform's reputation from the influence of formal cues, information on Twitter is often considered less credible than on other online news platforms news media (Schmierbach \& Oeldorf-Hirsch, 2012). However, as Twitter has recently come into wider usage in academic contexts (Collins et al., 2016), an updated study focusing on the credibility of scientific information on Twitter is needed.

Despite the recency of the movement, academic twitter has already adopted several conventions in the composition of tweets concerning scientific findings. Tweets about science exhibit a high incidence of 
47 linking URLs, and tweets also frequency employ "mentioning" (including other's user ids) as another way

48 to refer outwards to additional information or support (Büchi, 2016; Schmitt \& Jäschke, 2017).

49 Furthermore, since scientists frequently use Twitter to promote new publications (Holmberg et al.,

50 2014), several features of the scientific paper that are readily transferrable to tweets, such as the title,

51 abstract, and figures, are formal cues that may contribute to credibility. Outside the fringes of discourse,

52 the public sees scientists as trusted sources of reliable scientific information such that trust in scientists

53 may "spill over" into higher rates of trust for science-specific media (Brewer \& Ley, 2013). For known

54 scientists to personally endorse science information, even by briefly acknowledging their authorship or

55 positive evaluation of the information, may similarly confer a boost to that information's credibility. It

56 may also be possible that formal cues that are strongly and widely associated with academic research,

57 such as scientific abstracts or figures, may also confer increased credibility for science information.

58 Social approval can strongly impact credibility evaluations (Kobayashi, 2018; Winter \& Krämer, 2014)

59 indicators of reception may also positively correlate with perceived credibility. Most social media

60

61

62

63

64

65

66

67

68

69

70

platforms have some means for users to rate content (e.g., "Like", "Favorite" and "Share") and to view aggregated ratings of others - these indicators of social approval may impact perceptions of credibility.

In this study, we used online surveys through Amazon's Mechanical Turk (AMT) to examine the perceived credibility of scientific information appearing across several popular online media platforms, with a focus on Twitter. We explored both the relative credibility of Twitter compared with other platforms and the extent to which various formal and textual features within Twitter are related to the perceived credibility of scientific information. We focus on Twitter because Twitter is widely used for the dissemination of science information (Mohammadi et al., 2018), and it has higher rates of participation for scientists than other social media platforms, even as the platform is beset with concerns over the credibility of its content (Shao et al., 2018; Vosoughi et al., 2018). Amidst these concerns, this study will offer a more concrete evaluation of Twitter's credibility compared with other platforms commonly used 
71 for disseminating science information. While the primary goal of this study is a descriptive

72 understanding of the features that contribute to information's perceived credibility on Twitter, the

73 results of this study will also inform recommendations for composing tweets about scientific papers that

74 are more likely to be perceived as credible.

\section{2. Methods}

76 We conducted a study in two parts. In Part One, we conducted a survey through Amazon's Mechanical

77 Turk (AMT) investigating user credibility evaluations of real-world instances of media coverage in Twitter

78 compared to four other online platforms: Online Video, Blogs, News Articles, and Scientific Abstracts.

79 The results of this survey were used to assess the extent to which the perceived credibility of

80 information on Twitter differs from that of other common platforms. In Part Two, we conducted a

81 survey that asked respondents to rate the credibility of artificially-constructed tweets. The results of this

82 survey were used to determine the extent to which a tweet's perceived credibility was related to its

83 formal cues.

\section{$84 \quad 2.1$ Material selection.}

85 Part One compared the degree of perceived credibility of tweets against other forms of media. We used

86 Altmetric.com, a major altmetric data aggregator, to identify and sample journal articles that appeared

87 at least once in a news site, a blog, on Twitter, and in an online video. Out of these media types, only

88 Twitter is a specific branded platform; however because each type represents a specific genre with

89 stylistic and formal conventions, we will refer to each source as a "platform" that is hosting and

90 presenting the scientific information. We used purposive sampling to identify five articles across a range

91 of topics. These studies and their abbreviated labels are provided in Table 1.

92 At the time of sampling, relatively few articles were covered on all four platforms; this is an admitted

93 limitation of the present study and is further addressed in our discussion. In total, we had 25 unique 
94 topic/platform combinations. For each of the text examples (Twitter, abstract, news, and blog) a

95 screenshot was taken with no modification. However, given the heterogeneity of the sources, the

96 screenshots were cropped in order to remove varying contextual influences and identifying information

97 as well as to enforce a similar size and proportion was across all screenshots. We maintained all titles,

98 bylines, and some pictures to keep the sources in their original form. Examples of screenshots for one

99 study is provided in Fig 1.

Table 1. Abbreviated labels given to studies and Statements of Fact used in the Part One survey

Label

\begin{tabular}{l|r|r}
\hline Caffeine & $\begin{array}{r}\text { Post-study caffeine administration enhances memory } \\
\text { consolidation in humans }\end{array}$ & (Borota et al., 2014)[24] \\
\hline Dogs & Dogs are sensitive to small variations of the Earth's \\
magnetic field & (Hart et al., 2013)[25] \\
\hline Sweeteners & Artificial sweeteners induce glucose intolerance by \\
& altering the gut microbiota & (Suez et al., 2014)[26] \\
\hline Vitamins & Vitamin D and the risk of dementia and Alzheimer \\
& disease & (Littlejohns et al., \\
& 2014)[27] \\
\hline Marriage & Relationship between Wedding Expenses and \\
Marriage Duration & (Francis-Tan \& Mialon, \\
& 2015)[28]
\end{tabular}




\section{A. Scientific Abstract}

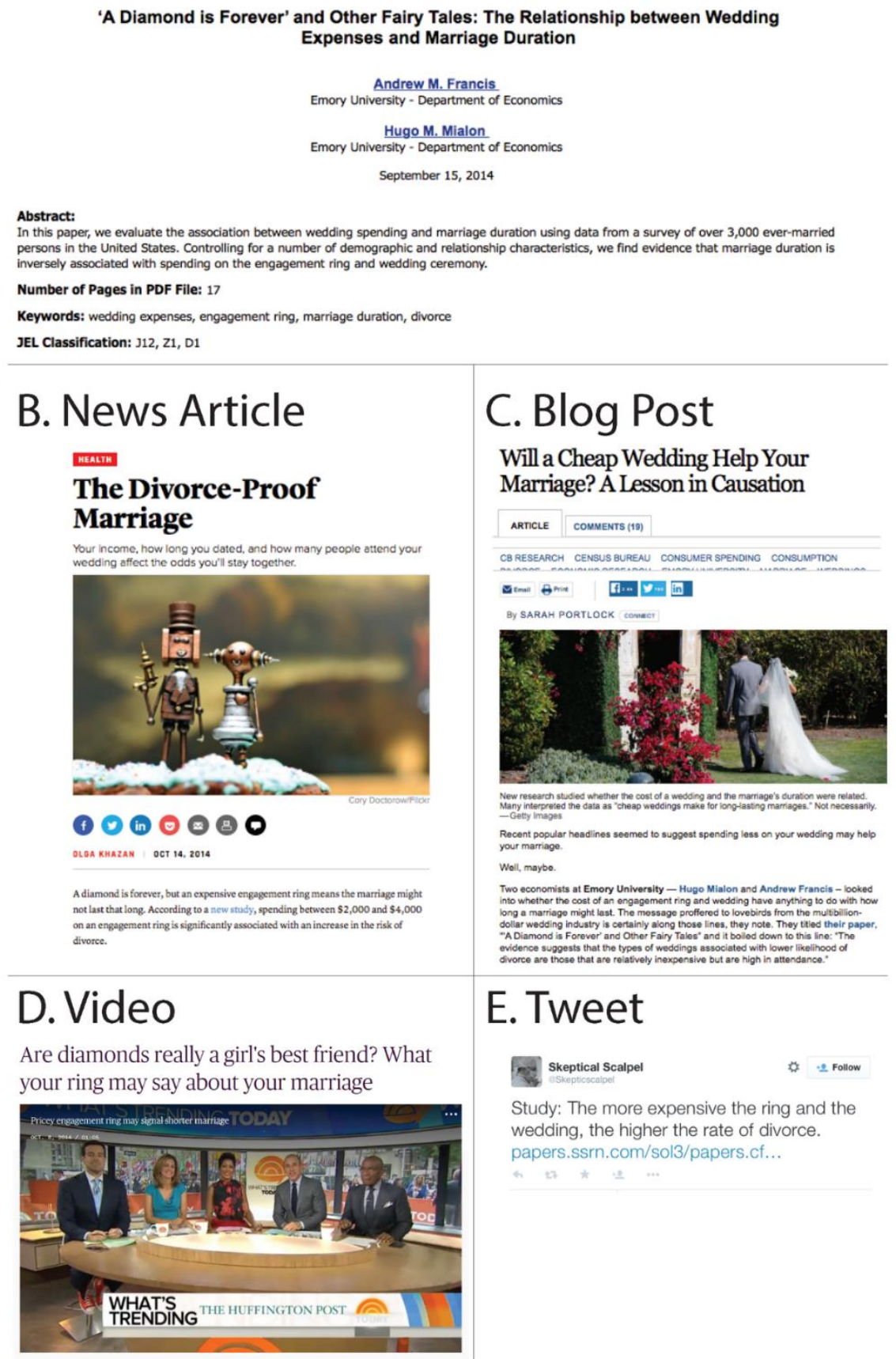

Fig 1. Examples of screenshots shown to survey respondents. All examples included in this figure relate to the journal article "Post-study caffeine administration enhances memory consolidation in humans" [24]. Listed are screenshots shown to respondents to assess the credibility of $(A)$ the journal abstract, (B) a news article, (C) a blog post, (D) an online video (respondent would view the entire video); and (E) a tweet about the information in the study. Access to all screenshots used in the study is available at https://github.com/murrayds/. 
Given that Twitter has been identified as a resource for paper sharing among academic scientists

101 (Mohammadi et al., 2018), Part Two of the study focused exclusively on identifying features in tweets

102 that were most strongly associated with high perceived credibility. Our sample was drawn from a s

103 single field-specific journal, the Annual Review of Clinical Psychology, in order to control for the field

104 differences observed in Part One. The labels given to each of the studies included in Part Two are listed

105 in Table S1.
A. Claim of
ownership
Claim of ownership
Our latest publication! Conducting Clinica Research Using Crowdsourced Convenience Samples bit.ly/2khkkPS

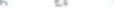
No claim of ownership
Conducting Clinical Research Using Crowdsourced Convenience Samples bit.ly/2khikkPS
B. Reception
Low reception
Our great study about potential problems in using risk assessment in criminal sentencing using risk ass
bit.ly/2, INVZIY
Medium reception
Our great study about potential problems in using risk assessment in criminal sentencing bit.ly/2jNVZjY
Our great study about potential problems in using risk assessment in criminal sentencing bit.ly/2]NVZIY
High reception

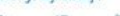

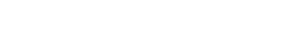
เ2 16 * 23
Q 2705201

\section{Phrasing of title

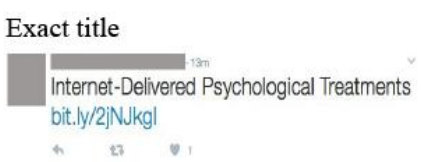
Positive paraphrase
Our great study about the importance and use of Internet-delivered psychological treatments bit.ly/2jNJkg
Neutral paraphrase

\section{Presence of visual}
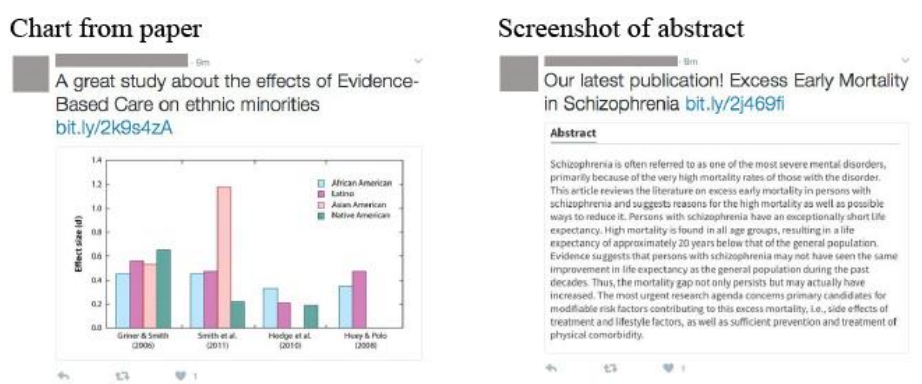
No visual
Our latest publication! The Efficacy of Exposure Therapy for Anxiety-Related Disorders and Its Underlying Mechanisms bit.ly/2kdnksk

Fig 2. Example of tweet screenshots shown to survey respondents. Twelve tweet variants were constructed for each of the 14 studies used in the second survey. Each constructed tweet contained a combination of features relating to (A) whether there was a claim of ownership, (B) its audience reception. (C) the phrasing of its title, and (D) the presence of a visual. Username and profile images were removed. Because only twelve tweets were created for each study, not all possible tweet variants exist for every study; instead, each study only contains twelve of the possible feature combinations, organized such that all features are equally represented.

106 We constructed a series of sample tweets based on each article, with varying combinations of the

107 following characteristics: 1) Use of first person possessive language like "our" to indicate authorial 
ownership of the paper's findings; 2) Low, medium, or high tweet reception numbers in the form of likes

109 and retweets; 3) Use of verbatim paper title, neutral paraphrase, or positive paraphrase; and 4) the

110 presence of a figure from the selected paper, its abstract, or no visual. We gave the impression that

111 identifying details, such as a Twitter handle or profile picture, were removed by placing a grey box

112 where this information should appear. Each tweet contained a URL to its corresponding journal article as

113 it common for science tweets (Büchi, 2016), but tweets were presented as images and so the link was

114 not clickable. All tweet screenshots are included in the supporting material of this study. Examples of

115 constructed tweets are provided in Fig 2.

\section{$116 \quad$ 2.2 Survey: Part One}

117 We sought user credibility judgements using Amazon's Mechanical Turk (AMT) service, a crowd-sourcing

118 application where respondents are solicited to perform tasks in exchange for monetary payment.

119 Drawing survey participants from Amazon's Mechanical Turk is a common research procedure that is

120 accepted to be a reputable means for recruiting a diverse set of survey participants in the current

121 survey-weary climate (Cassese et al., 2013; Sheehan \& Pittman, 2016).

122 All responses to Part One of the survey were obtained on June $16^{\text {th }}$ and $17^{\text {th }}, 2015$. Five distinct Human

123 Intelligence Tasks (HITs) were generated in AMT. Each HIT was a set of five distinct topics from each of

124 the five platforms, which ensured that topic and platform was presented to each participant. While the

125 embedded video needed to be presented last due to technological constraints, the other platforms and

126 the topics were presented to respondents in several shuffled sequences. A pilot test was conducted with

127 two participants that was used to refine the presentation of the HIT. The recruitment material is

128 presented in Text SI.1. We asked those who had completed one of the previous News Credibility HITs to

129 not complete another because, once exposed to the five topics, the respondent's impressions of

130 credibility and familiarity would likely be compromised. Even though a participant could theoretically

131 look up the author or articles, they were not directly linked to the sources. 
Title: News credibility

Description: You will be shown a variety of news sources on a variety of topics. You will be asked to assess the credibility of these sources, as well as your familiarity with the topics.

Keywords: news, classification

Instructions: The following HIT is designed to assess the degree to which you believe that a statement is credible. Please read/view all materials carefully. The results of this research will not be used to identify you.

"NOTE: If you have already completed one of these News Credibility HITs, do NOT complete any further HITs. Your work will be rejected if you do!"

\section{Fig 3. Presentation of HIT.}

Once the respondent accepted a HIT, they were presented with a series of screenshots followed by a video for the five topics. While the video was presented last due to the need to embed it, the order of the screenshots was randomized. In addition, a statement corresponding with the topic was presented. As with the topics that were chosen, these statements were limited by the need for them to appear clearly in all five platforms for each topic. They were intended to be simple so that it was clear they directly corresponded to the finding of each original article. The statements of fact corresponding to each article were as follows in Table 2.

Table 2. Statements of Fact for Each topic

Topic Label

Statement of Fact

\begin{tabular}{l|r|}
\hline Caffeine & Caffeine can improve memory. \\
\hline Dogs & Dogs are sensitive to Earth's magnetic field. \\
\hline Sweeteners & Artificial sweeteners may lead to diabetes. \\
\hline Vitamins & Vitamin D deficiency causes an increased risk of dementia and Alzheimer disease. \\
\hline Marriage & Marriage duration is inversely associated with spending on the engagement ring \\
&
\end{tabular}

Following the statement, participants were asked the following in the survey interface:

1. Based on this source, how credible do you find the following statement on a scale of 1-7, with 1 being "not at all credible" and 7 being "very credible"?

2. Before viewing this HIT, how familiar were you with this topic on a scale of 1-7, with 1 being "not at all familiar" and 7 being "very familiar"?

137 After responding to the five topic/genre combinations, respondents were asked to provide basic 
139 from one of "Male", "Female", or "Other". Age was entered freely as an integer. Location was selected 140 as either from the United States or not. Users selected their highest education earned from a list of 141 seven categories including "less than high school”, "high school”, "Associate", "Bachelors", "Masters",

142 "Doctorate", or "Professional"; to simplify analysis this variable was later collapsed into a binary variable 143 indicating whether or not the respondent had obtained a university degree (Bachelors, Masters, 144 Doctoral, or Professional). Each of these questions allowed for no answer to be provided, which would 145 result in a missing value. Respondents were also offered a free-input box to detail any issues that they 146 had with reading the textual excerpts or viewing the videos (which were hosted on YouTube or 147 embedded in news websites).

148 HITs were sent in batches over the course of the two days to recruit sufficient numbers of participants 149 with no duplication. Respondents were initially paid $\$ 0.10$ per HIT. However, given the length of time 150 that it took to complete the HIT this was increased to $\$ 0.25$ per HIT for the final batch. A validation 151 question ("What is two plus two?") was added to detect participants who were answering questions 152 randomly or otherwise not paying full attention to the task.

153 After removing records of respondents who failed the validation question and duplicate respondents (in 154 which case the respondent's first response was kept and all later responses excluded), the final dataset 155 consisted of 4,601 responses from 924 respondents. Most respondents for Part One, 56.0 percent, were 156 male $(n=517)$, and 2.9 percent $(n=27)$ selected either "other" or provided no gender. The average age 157 of a respondent was 33 years, whereas the median age was 30 years old. The majority, 58.7 percent, of 158 respondents reported having a university degree or higher $(n=542)$. The majority of respondents, 90.8 159 percent, reported their location as within the United States $(n=839)$. 


\subsection{Survey: Part Two}

In order to assess the relationship between tweet characteristics and perceptions of credibility, we performed a second survey with Amazon's Mechanical Turk using artificially constructed tweets (see the Materials section) on December $15^{\text {th }}, 2017$. The description of the new HITs read as follows: "You will be shown a variety of tweets on a variety of topics. You will be asked to assess the credibility of these tweets." Participants were paid $\$ 0.25$ for completing one HIT and were prohibited from responding to more than one HIT using the Unique Turker script (Ott, 2016).

Participants were shown 14 tweet variants on each of the 14 distinct topics. While the order of the topics remained constant, from topic 1 to topic 14 , the variations and combinations of tweet features was shuffled for different respondents. For each tweet, the participants were instructed to answer, "How credible do you find this tweet on a scale of 1-7, with 1 being "not at all credible" and 7 being "very credible"?" A series of demographic questions were posed at the end of the HIT that were presented and processed in the same fashion as for Part One. A validation question (i.e., "What is $2+2$ ?") was also asked, and a comment box was provided. The most frequent comments related to the size and/or resolution of certain tweets, which participants reported made it more difficult to ascertain their credibility.

After removing records of respondents who failed the validation question the final dataset for Part Two consisted of 1,568 responses by 112 respondents. The demographics of Part Two survey respondents was similar to those of Part One, though with fewer U.S. respondents and more having a university degree or higher. The majority of respondents for Part Two, 55.4 percent $(n=62)$ identified themselves as male, and three either reported their gender as "other" or did not report a gender. The average age of a respondent was 35 years, whereas the median age 32 years old. The majority, 69.9 percent, of respondents reported having a university degree or higher $(n=78)$. The majority of respondents, 75.9 percent, reported their location as within the United States $(n=85)$. 


\section{$184 \quad 2.3$ Analysis.}

185 For Part One, we used the data from the first survey to investigate the respondent's views of a statement's credibility and its relationship to the platform of presentation. The relationship between stated credibility and platform was assessed with a linear mixed effects model with the worker ID of the survey respondents as the random intercept in order to account for variability between respondents, implemented using the R package Ime4 (Bates et al., 2015). In the resulting regression, the stated credibility (an integer from one to seven) was used as the response variable and the platform as a

191 predictor variable. The predictor platform was a categorical variable for which "abstract" was set as the 192 reference level. The topic of study was included as a control variable and represented as a categorical 193 variable for which "Caffeine" (see Table S2) was set as the reference level. We also controlled for the 194 respondent's stated familiarity with the topic (integer from one to seven). Other control variables 195 included the gender, age (in decades), education level, and location of the respondent. Records with 196 missing values accounted for only about 3.4 percent of total responses and were excluded from the 197 regression analysis. We followed this regression with a series of univariate analyses that were used to 198 investigate the extent to which the distribution of and mean credibility differed between combinations 199 of the platform of presentation and topic of the study.

For Part Two, using the data from the second survey we conducted another linear mixed effects model

201 with the worker ID as the random intercept to assess the extent to which the characteristics of tweets

202 related to their perceived credibility. A regression analysis was used in which the stated credibility (an

203 integer from one to seven) was used as the response variable and the characteristics of the tweet as the 204 predictor variables. Four predictor variables were used to characterize each tweet. These included 1) a 205 binary variable indicating whether the tweet's author claimed ownership of the study; 2) a categorical 206 variable indicating whether the tweet contained no visual (reference level), a figure from the paper, or a 207 screenshot of the abstract; 3) a categorical variable indicating whether the tweet had a low (reference 
level), medium, or high reception, characterized by the number of likes and retweets (see examples in

209 supplemental materials); and 4) a categorical variable indicating whether the tweet included the title of

210 the paper (reference level), a positive paraphrase of the title, or a negative paraphrase of the title. We

211 controlled for topic of the study which was represented as a categorical with 14 levels, of which the first

212 was held as the reference (Table S4). Other control variables included respondent demographics which

213 were coded in the same way as in the first regression in Part One. The three respondents who did not

214 report a gender were excluded from the second regression analysis.

215 All data pre-processing and analysis was carried out using the programming language R 3.5.1 and

216 RStudio version 1.1.46. Code and anonymized data to replicate this analysis have been made available at 217 https://github.com/murrayds/sci-online-credibility.

\section{3. Results}

219 The object of this study has been the relationship between the perceived credibility of science

220 information and the platform on which it was presented, and the extent to which several language and

221 formal features on a salient platform, Twitter, contributes to perceived credibility. In this section, we

222 present the results of the analyses of participant responses indicating perceived credibility of science

223 information from two surveys, the first on perceived credibility on five different online platforms and

224 the second on different presentations within Twitter, one of the salient platforms in Part One.

225 Using an analysis of the Part One survey, we addressed the extent to which science information will be

226 credible on Twitter compared to science information on other platforms. As shown in Fig 4, all topics

227 were associated with lower credibility than the "Caffeine" study (see Table 1 for the terms that were

228 used for each study topic) with the "Dogs" topic being the least credible ( $\beta=-0.69, \mathrm{Cl}=[-0.82,-0.55])$,

229 followed by "Marriage", $(\beta=-0.57, \mathrm{Cl}=[-0.71,-0.44])$; the credibility of the "Vitamins", $(\beta=-0.047, \mathrm{Cl}=$ 
$230[-0.18,0.09])$, and "Sweeteners" ( $(=-0.029, \mathrm{Cl}=[-0.17,0.10])$ topics were only slightly lower than the

231 "caffeine" topic. The respondent's stated familiarity with the topic was the largest positive predictor of

232 credibility $(\beta=0.23, \mathrm{Cl}=[0.20,0.25])$. Compared to female respondents, being male was associated with

233 lower perceptions of credibility $(\beta=-0.076, \mathrm{Cl}=[-0.19,0.04])$ whereas a gender of "other" or "unknown"

234 was associated with even lower credibility, though with wide confidence intervals $(\beta=-0.31, \mathrm{Cl}=[-0.65$,

$2350.04])$. The age of respondents was trivially associated with increased perceived credibility $(\beta=0.0036$,

$236 \mathrm{Cl}=[-0.051,0.059])$. Compared to those with associates degrees or lower ("Associates-"), respondents

237 with a university degree or higher ("University+") were trivially associated with lower perceived

238 credibility $(\beta=-0.004, \mathrm{Cl}=[-0.12,0.094])$. A respondent being from the U.S. was associated with lower

239 perceived credibility $(\beta=-0.50, \mathrm{Cl}=[-0.71,-0.28])$.

240 Controlling for topic and respondent demographics, all platforms were associated with lower credibility

241 than journal abstracts. The platform with credibility scores most similar to journal abstracts were news

242 articles $(\beta=-0.77, \mathrm{Cl}=[-0.91,-0.64])$, followed by online video $(\beta=-0.78, \mathrm{Cl}=[-0.92,-0.65])$, blogs $(\beta=-$

$2430.94, \mathrm{Cl}=[-1.08,-0.80])$, and finally tweets, which were associated with 1.4 points lower credibility than

244 abstracts $(\beta=-1.4, \mathrm{Cl}=[-1.54,-1.27])$. The ANOVA of the linear model (Table S3) revealed that the

245 platform accounted for the largest proportion of total variance, followed by the stated familiarity and

246 the topic of study. The demographics of respondents accounted for little of the total variance. 


\section{A. Model coefficients, Survey 1}

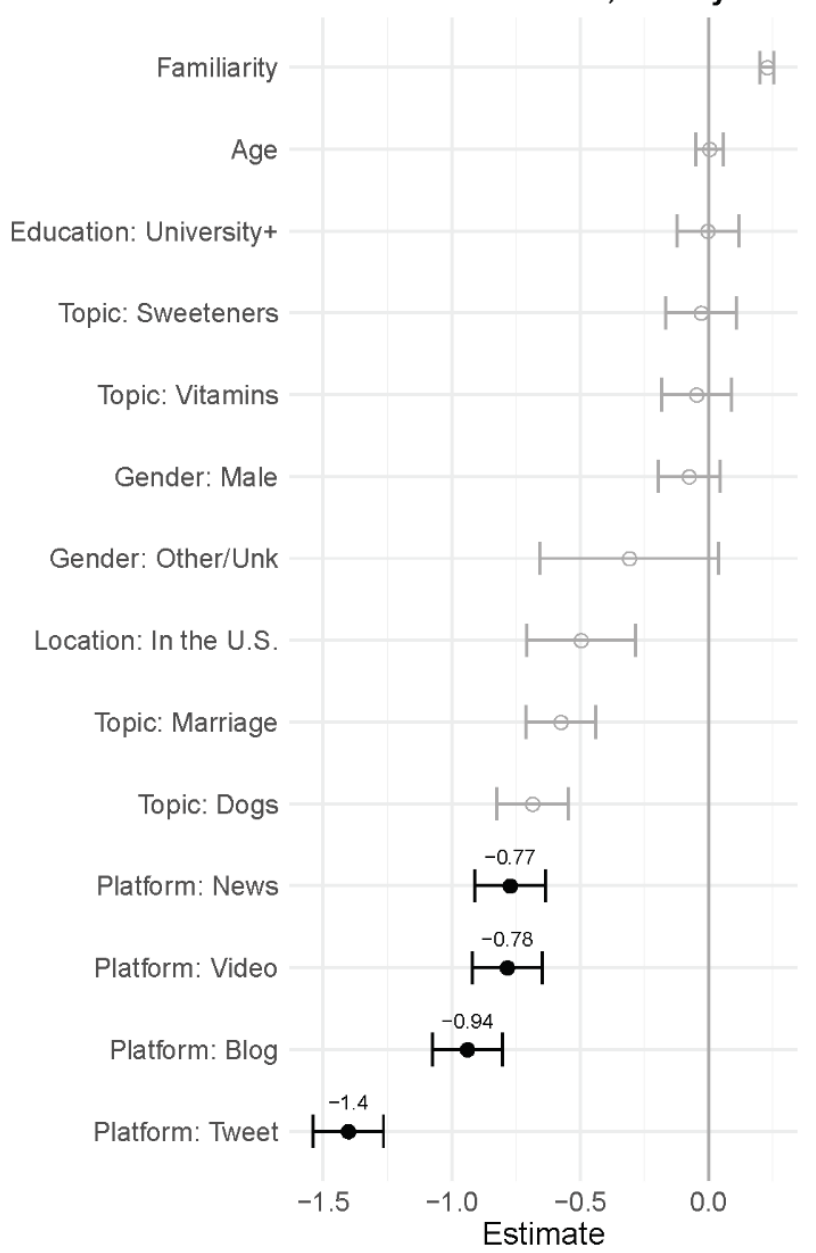

B. Credibility by medium

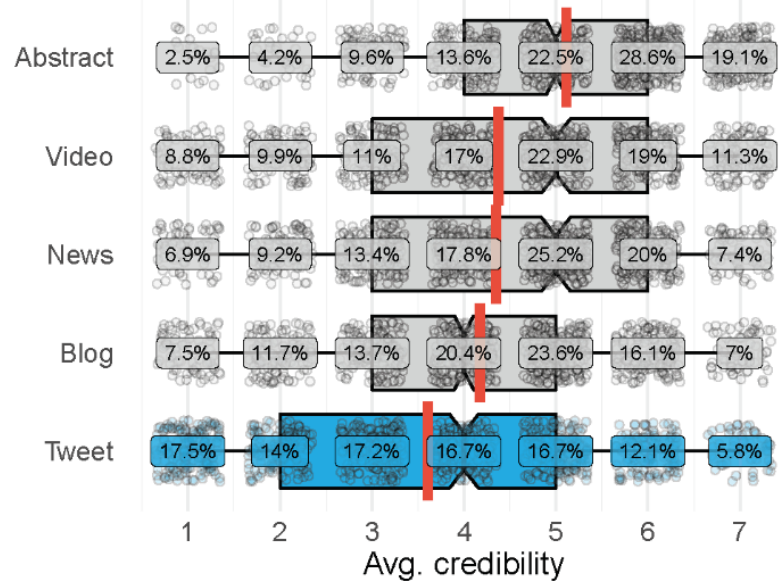

C. Response count by medium and topic

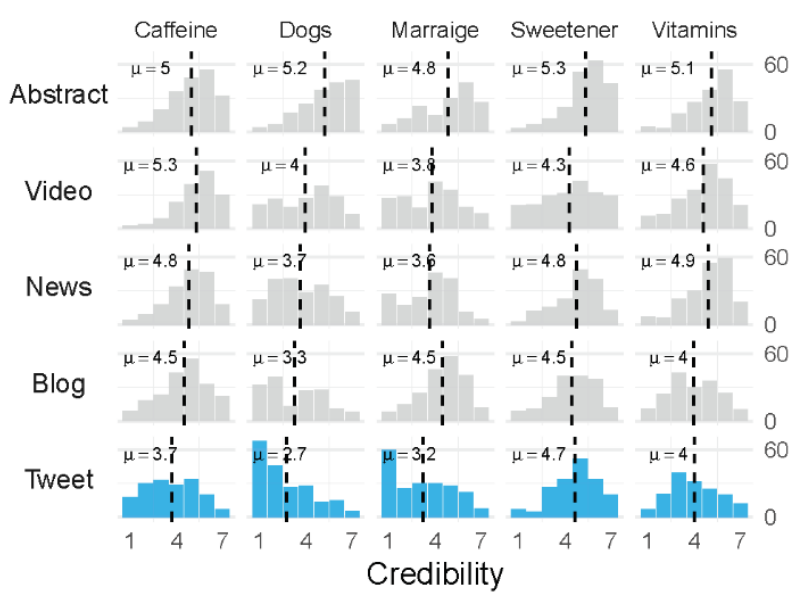

Fig 4. Abstracts are most credible and tweets are least credible. (A) Coefficients of mixed effects linear regression using the Credibility (1-7, treated as continuous) as the response variable. The $x$-axis corresponds to the coefficients for each predictor variable listed on the $y$-axis. Error bars correspond to the 95th percentile confidence intervals. The effects of predictor variable of interest, platform, have been highlighted. For Platform, the factor level "Abstract" has been held as the reference level. For Topic, "Caffeine" was the reference level. For Gender, "Female" was the reference level. For Education, "Associate-" (associates degree or lower) was the reference level. More detailed output can be found in Table S1. (B) Boxplots of the distribution of credibility scores for each platform. The notch corresponds to the median value, whereas the red line corresponds to the mean. Dots represent each observation scattered around each possible Credibility value. Labels refer to the number of responses for each credibility score as a proportion of all responses for that platform. (C) The distribution of responses for each Platform/Topic combination. The black dashed line refers to the mean of each distribution, which is also stated in the top left of each plot. 
249 lowest (median $=4$, mean $=3.61$ ) credibility ratings, 1.5 points of difference. Put another way, abstracts

250 were associated with around 40 percent greater credibility than tweets. Tweets also received the largest 251 proportion of one-point credibility ratings ( 17.5 percent) and had the lowest $25^{\text {th }}$ percentile rating of 252 two points.

253 While general patterns can be observed between platforms, there is also heterogeneity between topics,

254 (Fig 4). In all but one topic ("Caffeine") abstracts had the highest credibility on average. This is true even 255 for the "Vitamin" topic, for which respondents complained that the screenshot of the abstract was too 256 blurry to read. For all but one topic ("Sweeteners"), tweets had the lowest credibility ratings, with the 257 lowest overall for "Dogs": for this topic the distribution of ratings for tweets was nearly the inverse of 258 the abstract, the former received a plurality of one-point ratings whereas abstracts received a plurality 259 of seven-point ratings. It is more difficult to discern clear patterns among the remaining platforms, 260 though taken together, Figs 4A, 4B and 4C suggest that online videos and news articles received roughly 261 similar credibility ratings, whereas blogs tended to receive lower ratings than news outlets but higher 262 than tweets.

263 Tweets were associated with the lowest credibility ratings compared to all other platforms. However,

264 Twitter remains one of the most popular media platforms used by researchers (Piwowar, 2013;

265 Rowlands et al., 2011; Tenopir et al., 2013; Van Noorden, 2014). While researchers have little control 266 over the platform constraints of Twitter itself, there are possibly actions they can take to compose 267 tweets in a way that maximizes their perceived credibility. In the second part of this study we assessed 268 the extent to which formal cues of tweets related to their credibility. Specifically, whether source cues 269 indicating a closer association with the scientists or the scientific process will be associated with higher 270 perceived credibility, and whether indicators of reception positively correlate with perceived credibility. 
271 In Fig 5, we present the results of a multiple linear regression applied to the responses for Survey Two,

272 using the credibility rating as the response and tweet characteristics, topic, and respondent

273 demographics as predictor variables. Topic 1 was held as the reference level, and compared to this

274 reference, all topics were associated with higher credibility except for topic 2 , which was trivially

275 associated with lower credibility. The topic associated with the highest credibility was topic 14 , which

276 was associated with a 1.9 point higher score than topic $1(\beta=1.9, \mathrm{Cl}=[2.6,3.2])$. The effects observed

277 when controlling for other variables differed from the univariate distribution of credibility by topic (see

278 Figure SI.2), for which topic 6 had the highest average credibility and topic 12 had the lowest. Male

279 respondents were trivially associated with higher perceived credibility ratings than female respondents

$280(\beta=0.049, \mathrm{Cl}=[-0.33,0.42])$. Older participants were also only trivially associated with lower perceived

281 credibility ratings $(\beta=-0.074, \mathrm{Cl}=[-0.24,0.096])$, and respondents with a university degree or higher

282 ("University+") were trivially associated with greater credibility ratings $(\beta=0.015, \mathrm{Cl}=[-0.42,0.45])$

283 when compared to those with associates degrees or lower ("Associates-"), . A respondent being from the

284 U.S. was associated with lower credibility ratings $(\beta=-0.30, \mathrm{Cl}=[-0.78,-0.17])$. All of these findings

285 reinforce those found in Part One. 


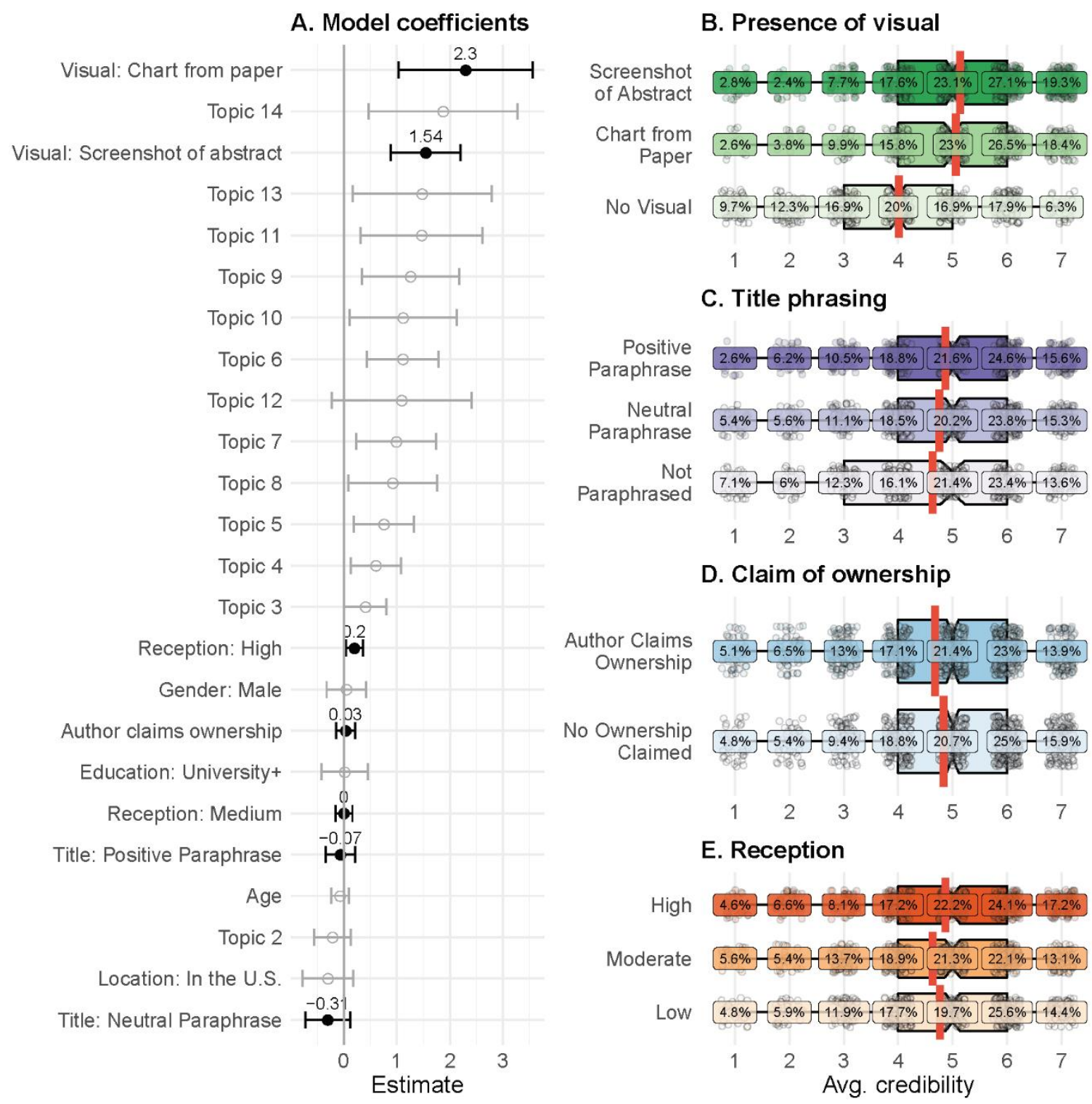

Fig 5. Charts and graphs associated with greater credibility. (A) Coefficients of linear regression using the Credibility (1-7, treated as continuous) as the response variable. The $\mathrm{x}$-axis corresponds to the coefficients for each predictor variable listed on the $y$-axis. Error bars correspond to the 95th percentile confidence intervals. The effects of predictor variable of interest, tweet characteristics, have been highlighted. For presence of visual, "no visual" was the reference; for title phrasing "no paraphrase" was the reference; for claim of ownership, "no claim" was the reference; for reception, "low" was the reference. For Topic, "Caffeine" was the reference level. For Gender, "Female" was the reference level. For Education, "Associate-" (associates degrees or lower) was the reference level. More detailed output can be found in Table S3. (B-E) Boxplots of the distribution of credibility scores across tweet characteristics for (B) presence of a title, (C) claim of ownership, (D) phrasing of the title, and (E) the reception of the tweet. The notch of the boxplot indicates the median value, whereas the red line indicates the mean. Dots represent each of the observations scattered around each possible Credibility value. Labels refer to the number of responses for each credibility score as a proportion of all responses for that feature. 
The tweet features indicating the presence of a visual accounted for the greatest total variance,

287 followed by topic of the paper (see the ANOVA in Table S5). Controlling for topic and respondent 288 demographics, tweets that contained a chart from the paper were associated with a full 2.30 points 289 higher credibility rating than tweets that did not contain any visual $(\beta=2.30, \mathrm{Cl}=[1.0,3.6])$ which was 290 held as reference, even as the confidence interval was quite wide. Similarly, tweets that featured a 291 screenshot of the abstract were associated with 1.5 points higher credibility than those with no visual ( $\beta$ $292=1.5, \mathrm{Cl}=[0.88,2.2])$. Paraphrasing, both positive and neutral, was associated with lower credibility 293 than no paraphrasing -0.31 point lower for Neutral paraphrasing $(\beta=-0.31, \mathrm{Cl}=[-0.73,-0.16])$ and 2940.065 lower for positive paraphrasing $(\beta=-0.065, \mathrm{Cl}=[-0.34,-0.12])$-but only trivially as the confidence 295 interval for both conditions crossed 0. Claiming ownership in a tweet was associated with a trivially 296 greater credibility ratings than not $(\beta=0.032, \mathrm{Cl}=[-0.15,0.21])$. Medium reception tweets were not 297 associated with a strong increase or decrease in credibility fromlow reception tweets $(\beta=-0.00092, \mathrm{Cl}=$ $298[-0.16,0.16])$, but high-reception tweets were associated with slightly more credibility $(\beta=0.19, \mathrm{Cl}=$ $299[0.036,0.36])$.

300 We further investigated the distribution of credibility responses by each tweet characteristic. Fig 5B 301 demonstrates the extent to which tweets containing a visual, whether a chart (median $=5$, mean $=5.14$ ) 302 or a photo of the abstract (median $=5$, mean $=5.06$ ), were rated as more credible, on average, than 303 tweets with no visual (median $=4$, mean $=4.01$ ). Differences were less pronounced for title phrasing, 304 differing from effects observed in Fig 5A when controlling for topic and respondent demographics. Fig $3055 \mathrm{C}$ shows that, considering only the raw distribution, the average credibility for both positive (median = 3065 , mean $=4.87)$ and neutral $($ median $=5$, mean $=4.75)$ paraphrasing is higher than that of no 307 paraphrasing (median $=5$, mean $=4.63$ ). This may indicate that other factors, such as the title of the 308 study itself, have more to do with the perceived credibility of the tweet than the phrasing. The opposite 309 was true for claims of ownership - while claiming ownership was associated with increased credibility in 
4.A, claiming ownership is associated with slightly lower mean (median $=5$, mean $=4.68$ ) than not

311 claiming ownership (median $=5$, mean $=4.84$ ) in 4.C. For reception there were no notable differences

312 between the regression and raw distribution; compared to tweets with a low reception (median = 5,

313 mean $=4.76)$, tweets with moderate reception were associated with slightly lower (median $=5$, mean $=$

314 4.64) and tweets with high reception with slightly higher credibility (median = 5, mean = 4.87).

\section{4. Discussion}

316 Researchers have been encouraged to engage with a wider audience with their research, and online

317 platforms offer a prime avenue through which they can reach broad interested audiences. However,

318 online platforms are often crowded with both accurate information and disinformation. In order to cut

319 through noise, researchers and science communicators need a better understanding of how the

320 platform and composition of their content relate to its perceived credibility. Research on credibility

321 emphasizes the role of formal cues that users draw upon as they evaluate unfamiliar subject matter

322 (Lucassen \& Schraagen, 2013). Even within the limited space of Tweets, the inclusion of particular formal

323 cues are associated with higher credibility of science information.

324 We observed evidence consistent with past studies that Science information is least credible on Twitter

325 compared to science information on other platforms (Schmierbach \& Oeldorf-Hirsch, 2012). Twitter was

326 rated the least credible platform, even when controlling for the topic, respondent's stated familiarity,

327 and respondent demographics. While there was heterogeneity by study, Twitter was rated as least

328 credible on average, for four of the five study topics. Differences in platform have been shown to

329 influence user perceptions of credibility to the point that such associations have been framed as bias in

330 previous studies (Schmierbach \& Oeldorf-Hirsch, 2012; Winter \& Krämer, 2014). Research on credibility

331 emphasizes the role of formal cues that users draw upon as they evaluate unfamiliar subject matter

332 (Lucassen \& Schraagen, 2013). The particular formal cues of Twitter, such as character restrictions that 
333 leave little space for the inclusion of cues that could signal credibility, may affect the overall credibility of 334 information on Twitter.

335 In contrast to tweets, scientific abstracts were consistently rated as the most credible, associated with 3360.77 more points from credibility ratings from the second most credible source, news articles, and 1.4 337 point (tweets) higher credibility ratings than tweets. The images of abstracts may impart some degree 338 of credibility to a claim. This notion was supported by an accidental finding from our analysis: after 339 receiving the survey results, we found that that the screenshot of the abstract for "Vitamin" study was 340 blurry and mostly illegible (Fig S3 A). Even though it was unreadable, the abstract was still rated more 341 credible than other platforms. The association of the abstract with the institution of science may bolster 342 its credibility, as was theorized by Brewer and Ley in their study of public perceptions of climate science 343 (Brewer \& Ley, 2013). The high credibility of the blurry abstract suggests that the format of the abstract 344 and its close association with the institutions of formal research could be more important to credibility 345 than the content itself.

346 Patterns for the remaining platforms were less clear. Generally, abstracts were rated as most credible, 347 followed by news articles and online videos, which tended to have similar credibility ratings. Blogs were 348 rated as one of the least credible platforms, second only to tweets. The confidence intervals calculated 349 for these three variables in the regression analysis all overlap, suggesting a lack of certainty in this 350 ordering (see Fig $4 \mathrm{~A}$ ). However, tweets were rated as the least credible with confidence intervals that 351 overlapped with no other platforms. This low credibility may not necessarily undermine Twitter's 352 usefulness for scientists performing outreach and professional networking; these findings only sound a 353 warning note that the deliberate use of appropriate formal and stylistic features to convey credibility is 354 important when composing tweets. 
355 We observed only partial support for the idea that formal cues in tweets that indicate a closer

356 association with the scientists or the scientific process will be associated with higher perceived

357 credibility. Specifically, we found that only one feature of a tweet, the presence of a visual in the tweet,

358 was significantly associated with increased ratings of credibility. The inclusion of a figure from the paper

359 was associated with highest credibility, and the inclusion a screenshot of an abstract than tweets as

360 nearly as high. However, as there was still considerable variability in these effects as seen in the wide

361 confidence intervals, the type and information density of the paper figures included in the tweet may

362 have a strong influence on the credibility. While the overall formatting of the scientific figures was in a

363 similar style as the figures originated from the same journal, the figure type varied from study to study

364 and included circle charts, schematic diagrams, bar graphs, and tables. Since abstracts and scientific

365 figures have strong associations with formal science, they may also appear especially credible. Future

366 work should investigate the effects of the inclusion of a generic image that is not the abstract or a figure

367 to investigate whether scientific figures in particular contribute to credibility, or if any image confers a

368 similar credibility boost. The remaining features that we observed were at most weakly associated with

369 credibility. There was some evidence that paraphrasing the title and claiming ownership relate to the

370 tweet's perceived credibility. However, since these effects disappear when other variable are controlled,

371 it is possible that they are artefacts of the papers themselves, rather than of the composition of the

372 tweet.

373 We observed only weak evidence that indicators of reception will positively correlate with perceived

374 credibility. Compared to tweets with low reception (low number of likes and retweets), tweets with

375 moderate reception were trivially-associated with lower credibility. However, tweets with high

376 reception were associated with slightly higher credibility. While this effect was found to be statistically

377 significant, its confidence intervals nearly included zero and its estimated effect was small, associated

378 with only 0.19 point greater credibility than low reception (Fig 5A; Table S4). Our finding regarding 
reception disagrees somewhat with past studies, which found that for other online formats, the presence of peer-evaluations was strongly related to credibility (Kobayashi, 2018; Winter \& Krämer, 2014). This discrepancy may result from differences between Twitter's formal cues and those of other platforms, if likes and retweets on Twitter are not interpreted by viewers as social approval, or if that

383 social approval is not as important to evaluating the credibility of information on Twitter in other contexts. Twitter's reputation for spreading misinformation (Shao et al., 2018; Vosoughi et al., 2018) may also lead users to become indifferent to reception.

We note several limitations to this study. For Part One of the study, articles were selected which appeared across several existing platforms, which was a high standard for inclusion; this meant that the five studies we selected were covered online due to their attention-grabbing qualities and may not be representative of scientific information as a whole. Variance in the presentation of topics across each platform is also a limitation; for example, the title of the "Dogs" news article was sensationalized as "Scientists observe dogs relieving themselves, discover something amazing", whereas the scientific 392 article was more neutrally-titled with "Dogs are sensitive to small variations in the earth's magnetic field." These differences in tone may have affected the users' evaluations of credibility for these articles.

394 There were issues with some screenshots that were discovered after the survey was conducted; for 395 instance, the abstract for the study about Vitamin D and reducing the risk of Alzheimer's was blurry. 396 Furthermore, only the Francis and Mialon 2015 paper showed the authors' institutional affiliation, which 397 could have affected the abstract results. More generally, there was variation in many factors in the 398 screenshots used to represent each platform. The use of real-world examples of online science 399 information lent realism to the study, but it also introduced many potential confounding factors such as 400 differences in formatting, presence of images, and the presentation of authors.

401 In Part Two of our study, we mitigated this heterogeneity by selecting articles from a single psychology 402 journal, but this may affect the generalizability of our findings if factors relating to tweet credibility for 
psychological sciences are not consistent across scientific fields. Furthermore, as the study topics of the

404 tweets in Part Two were presented in a consistent order, it is inadvisable to draw any conclusions about 405 the relative credibility of the topics due to order effects. The use of Amazon Mechanical Turk, rather 406 than other traditional survey methodology, makes it difficult to assess the representativeness of our 407 respondents and also limits the generalizability of our findings. In addition, real Twitter users are more 408 likely to know the authors of tweets they encounter, whether personally or by reputation, and this may 409 also contribute to tweet credibility that we have been unable to represent within the survey. However, 410 because many tweets that Twitter users are exposed to have been retweeted from users whom they do 411 not follow, we believe that perceived credibility in the absence of a pre-existing relationship between

412 users is important in itself. Despite these limitations, we observed clear and consistent trends that lend 413 insight into the factors influencing online credibility of scientific information.

\section{5. Conclusion}

415 In this study, we conducted a survey to investigate the extent to which perceived credibility of scientific 416 information differed between five online platforms. We observed that journal abstracts were

417 consistently deemed most credible whereas tweets were consistently deemed the least credible, though

418 we also noted high variance by topic. We then conducted a second survey in which we assessed the 419 extent to which perceptions of the credibility of tweets related to the characteristics of their 420 composition. We constructed a set of artificial tweets reporting science information but each with a 421 unique combination of key characteristics, including whether the tweet displayed an image relating to 422 the paper, the paraphrasing of the paper's title, whether the author claimed ownership of the research, 423 and the tweet's reception (number of likes and retweets). We observed that the presence of a chart or a 424 screenshot from the paper was associated with greater credibility but little to no evidence of meaningful 425 differences based on other factors. 
426 As researchers continue to leverage these platforms, it becomes important that researchers compose

427 scientific information in ways that make it most credible. Beyond helping researchers to communicate

428 their work, composing credible content may help the general public focus on the most credible scientific

429 information, rather than the wealth of disinformation. During outreach efforts, it is important that

430 researchers be cognizant of how context and format affect the credibility of the information they

431 present. Some formal cues are more influential than others in user's evaluations of credibility. Our

432 findings provide evidence of the importance of platform and topic to perceived credibility online. We

433 also demonstrated the importance of visuals to the perceived credibility of tweets. More work is needed

434 to understand how the credibility of scientific information differs by context and platform, and the ways

435 that the formal cues of information impact its credibility.

\section{Acknowledgements}

We would like to thank the members of our research group who gave useful feedback on a presentation of this work, and for useful discussions during our weekly meetings. 


\section{References}

Bates, D., Mächler, M., Bolker, B., \& Walker, S. (2015). Fitting Linear Mixed-Effects Models Using Ime4. Journal of Statistical Software; Vol 1, Issue 1 (2015). https://www.jstatsoft.org/v067/i01

Bian, B. (2012). Research of Factors on Impacting Internet Information Credibility Based on Electronic Commerce Users Demands. 2012 International Conference on Communication Systems and Network Technologies, Communication Systems and Network Technologies (CSNT), 2012 International Conference On, 987. edseee. https://doi.org/10.1109/CSNT.2012.210

Brewer, P. R., \& Ley, B. L. (2013). Whose Science Do You Believe? Explaining Trust in Sources of Scientific Information About the Environment. Science Communication, 35(1), 115-137.

Büchi, M. (2016). Microblogging as an extension of science reporting. Public Understanding of Science, 26(8), 953-968. https://doi.org/10.1177/0963662516657794

Cassese, E. C., Huddy, L., Hartman, T. K., Mason, L., \& Weber, C. R. (2013). Socially Mediated Internet Surveys: Recruiting Participants for Online Experiments. PS: Political Science and Politics, 46(4), 775-784. JSTOR.

Collins, K., Shiffman, D., \& Rock, J. (2016). How Are Scientists Using Social Media in the Workplace? PLOS ONE, 11(10), e0162680. https://doi.org/10.1371/journal.pone.0162680

Côté, I. M., \& Darling, E. S. (2018). Scientists on Twitter: Preaching to the choir or singing from the rooftops? FACETS, 3(1), 682-694. https://doi.org/10.1139/facets-2018-0002

Duggan, M., Ellison, Nicole. B., Lampe, C., Lenhart, A., \& Madden, M. (2015). Social Media Update 2014. Pew Research Center. http://www.pewinternet.org/2015/01/09/social-media-update-2014

Flanagin, A. J., \& Metzger, M. J. (2007). The role of site features, user attributes, and information verification behaviors on the perceived credibility of web-based information. New Media \& Society, 9(2), 319-342. https://doi.org/10.1177/1461444807075015

Holmberg, K., Bowman, T. D., Haustein, S., \& Peters, I. (2014). Astrophysicists' Conversational Connections on Twitter. PLOS ONE, 9(8), e106086. https://doi.org/10.1371/journal.pone.0106086

Kobayashi, K. (2018). The Impact of Perceived Scientific and Social Consensus on Scientific Beliefs. Science Communication, 40(1), 63-88. https://doi.org/10.1177/1075547017748948

Lucassen, T., \& Schraagen, J. M. (2013). The influence of source cues and topic familiarity on credibility evaluation. Computers in Human Behavior, 29(4), 1387-1392. https://doi.org/10.1016/j.chb.2013.01.036

McClain, C. R. (2017). Practices and promises of Facebook for science outreach: Becoming a "Nerd of Trust." PLOS Biology, 15(6), e2002020. https://doi.org/10.1371/journal.pbio.2002020

Mohammadi, E., Thelwall, M., Kwasny, M., \& Holmes, K. L. (2018). Academic information on Twitter: A user survey. PLOS ONE, 13(5), e0197265. https://doi.org/10.1371/journal.pone.0197265

Ott, M. (2016). Unique Turker Script. : https://uniqueturker.myleott.com/

Piwowar, H. (2013). Value all research products. Nature, 493(7431), 159-159. https://doi.org/10.1038/493159a

Ranger, M., \& Bultitude, K. (2014). 'The kind of mildly curious sort of science interested person like me': Science bloggers' practices relating to audience recruitment. Public Understanding of Science, 25(3), 361-378. https://doi.org/10.1177/0963662514555054

Rowlands, I., Nicholas, D., Russell, B., Canty, N., \& Watkinson, A. (2011). Social media use in the research workflow. Learned Publishing, 24(3), 183-195. https://doi.org/10.1087/20110306

Schmierbach, M., \& Oeldorf-Hirsch, A. (2012). A Little Bird Told Me, So I Didn't Believe It: Twitter, Credibility, and Issue Perceptions. Communication Quarterly, 60(3), 317-337.

https://doi.org/10.1080/01463373.2012.688723 
Schmitt, M., \& Jäschke, R. (2017). What do computer scientists tweet? Analyzing the link-sharing practice on Twitter. PLOS ONE, 12(6), e0179630. https://doi.org/10.1371/journal.pone.0179630

Shao, C., Ciampaglia, G. L., Varol, O., Yang, K.-C., Flammini, A., \& Menczer, F. (2018). The spread of lowcredibility content by social bots. Nature Communications, 9(1), 4787. https://doi.org/10.1038/s41467-018-06930-7

Shariff, S. M., Zhang, X., \& Sanderson, M. (2017). On the credibility perception of news on Twitter: Readers, topics and features. Computers in Human Behavior, 75, 785-796. https://doi.org/10.1016/j.chb.2017.06.026

Sheehan, K. B., \& Pittman, M. (2016). Amazon's Mechanical Turk for academics: The HIT handbook for social science research. Melvin \& Leigh, Publishers.

Tenopir, C., Volentine, R., \& King, D. W. (2013). Social media and scholarly reading. Online Information Review, 37(2), 193-216. https://doi.org/10.1108/OIR-04-2012-0062

Tseng, S. ( 1 ), \& Fogg, B. J. ( 2 ). (1999). Credibility and computing technology. Communications of the ACM, 42(5), 39-44. edselc. https://doi.org/10.1145/301353.301402

Van Noorden, R. (2014). Online collaboration: Scientists and the social network. Nature, 512(7513), 126129

Vosoughi, S., Roy, D., \& Aral, S. (2018). The spread of true and false news online. Science, 359(6380), 1146. https://doi.org/10.1126/science.aap9559

Winter, S., \& Krämer, N. (2014). A question of credibility - Effects of source cues and recommendations on information selection on news sites and blogs. 39. https://doi.org/10.1515/commun-20140020

Yammine, S. Z., Liu, C., Jarreau, P. B., \& Coe, I. R. (2018). Social media for social change in science. Science, 360(6385), 162. https://doi.org/10.1126/science.aat7303 